This is the author's accepted manuscript of an article that appears in : Social Care and Neurodisability published by Emerald. Please cite as:

Brian O'Neill, Catherine Best, Alex Gillespie, Lauren O'Neill, (2013)

"Automated prompting technologies in rehabilitation and at home", Social

Care and Neurodisability, Vol. 4 Iss: 1, pp.17-28

http://www.emeraldinsight.com/doi/abs/10.1108/20420911311302281

\title{
Automated prompting technologies in rehabilitation and at home
}

\section{ABSTRACT (250 words)}

Purpose: To test the efficacy of an interactive verbal prompting technology

(Guide) on supporting the morning routine. Data has already established the efficacy of such prompting during procedural tasks, but the efficacy of such prompting in tasks with procedural and motivational elements remains unexamined. Such tasks, such as getting out of bed in the morning and engaging in personal care, are often the focus of rehabilitation goals.

Design/methodology/approach: A single-n study with a male (age 61) who had severe cognitive impairment and was having trouble completing the morning routine. An A-B-A'-B'-A"'-B'design was used with the intervention phase occurring both in an in-patient unit (B, B') and in the participants' own home (B").

Findings: Interactive verbal prompting technology (Guide) significantly reduced support worker prompting and number of errors in the in-patient setting and in the participant's own home.

Research limitations: The results suggest that interactive verbal prompting can be used to support motivational tasks such as getting out of bed and the morning routine. This study used a single subject experimental design and the results need to be confirmed in a larger sample. 
Originality/value: This is the first report of use of interactive verbal prompting technology to support rehabilitation of a motivational task. It is also the first study to evaluate Guide in a domestic context.

\section{Introduction}

Cognitive impairment mediates disability in conditions where brain function is impaired. According to the International Classification of Functioning (ICF; WHO 2002), higher-level cognitive functions, or executive functions, underpin goal oriented behaviours, such as abstraction, planning, time management, cognitive flexibility, sequencing, problem solving and judgement. Deficits of higher-level cognitive functions are often catastrophically disabling (eg. Oddy and Worthington 2009) and thus require costly carer input. 
Assistive technology for cognition (ATC) is the use of technology to extend or augment mental functions, with particular application meeting the needs of people with cognitive impairment. The use of technology to extend human abilities is universal. Bows extend the ability to launch projectiles; knives augment the ability to bite and tear. It can be argued that the peculiar facility for the selection and assimilation of technology defines humanity (Aunger 2010; Clark 2003). In recent times, digital technology has matured to be sufficiently portable and capacious to further extend human cognitive function. Smart mobile phones support both communication across distances and prospective memory through reminders and calendar functions. Despite their ubiquity these tools have not been widely used by the people who have the most to gain, that is, people with impairment to their cognitive function. The learning and memory burden involved the use of early digital technologies is changing, with simpler more intuitive interfaces being developed. There is currently a burgeoning interest in the field of assistive technology for cognition, as reviewed in Gillespie, Best and O'Neill (2012). ATC is promising in its development of compensatory technologies to augment existing or preserved cognitive abilities and has potential to reduce disability and dependence. The portable or ambient technology can be thought of a prosthesis or replacement for the specific impaired cognitive function (Cole 1999).

Gillespie, Best and O'Neill (2012) found that a large proportion of ATC have been used to assist with time management (33 studies of 91 included studies) and organisation and planning (25 studies of 91). These domains are highly relevant to activities of daily living.

Time management functions are prospective memory functions that ensure that one behaviour stops and another begins at a specific time. For example, reminding the user to leave to go to a doctor's appointment at a specific time. Time management is the most common ICF specific mental function targeted by ATC. The largest study in the ATC field is the Neuropage randomised controlled trial (Wilson et al., 2001), $n=143$, which demonstrated the efficacy of using a paging system to deliver reminders for the performance of everyday 
tasks in people with cognitive impairments. The efficacy of pagers to perform this function has also been demonstrated (Kirsch, Shenton, \& Rowan, 2004). Other media have also used text or auditory prompts to overcome prospective memory difficulties. Voice recorders with a timer function (eg. van den Broek, Downes, Johnson, Dayus, \& Hilton, 2000); text messaging to mobile phones (Pijnenborg, Withaar, Evans, van den Bosch, \& Brouwer, 2007); voice messages to phones (Leirer, Morrow, Tanke, \& Pariante, 1991); smartphone reminders (Svoboda \& Richards, 2009) or schedule management software on a palmtop computer (Kim, Burke, Dowds Jr., Boone, \& Park, 2000) or PDA (eg. Davies, Stock, \& Wehmeyer, 2002) also have demonstrated efficacy. We would conclude that proof of concept is established and that for those with specific prospective memory difficulties, unidirectional text and recorded prompts to mobile devices are effective interventions.

Difficulty monitoring one's behaviour can lead to problems performing complex sequences. Successful sequence performance requires active maintenance of the goal state, most recent step, correct next step and solutions to problems arising. In contrast with the large number of studies with the aim of supporting organisation and planning functions, there have been only a limited number of ATCs developed which provide step-by-step support during task performance.

Lancioni et al. (2000) developed the VICAID system to be used by people with intellectual disability to guide them through domestic and vocational tasks. The VICAID system is a palm top computer with a simplified user interface consisting of a single button, providing visual and auditory prompts through tasks. Users provide feedback to the system by pressing the button. VICAID also rewards successful task completion through feedback to the user.

Mihailidis et al. (2008) developed the COACH system to prompt users with dementia through processes such as hand washing. The latest version of the device uses a camera to capture visual data on the position of the users' hands to enable the system to be context aware of the user's progress through the task and thus select the appropriate auditory prompt. 
The Guide system aims to emulate the verbal support provided by carers (O'Neill \& Gillespie, 2008). Guide provides verbal prompts to orient users to sub-steps, remind users to perform various checks, and work with users to resolve problems. Users respond to the prompts using the simple verbal responses of "yes" and "no." When loaded with a protocol mapping the action pathway and common problems, Guide is an expert system able to deal with problems that might arise. The system sequences the task for users in terms of sub-steps, and for each sub-step, a series of questions are asked. Affirmative responses lead to the next question or sub-step. Negative responses lead to problem solving sub-routines. Thus, relatively able users can move quickly through the protocol, while less able users receive more guidance. The contribution is in the close simulation of carer scaffolding of task performance. The auditory-verbal interface emulates conversational interaction; does not distract the user's visual attention from the task and can resolve commonly arising problems. Guide prevents errors because it prompts users before each action and thus implements an errorless learning approach.

O'Neill, Moran and Gillespie (2010) examined the use of Guide to aid a complex rehabilitation sequence (donning a prosthetic limb) in a sample of 8 older adults with cognitive impairment of vascular origin. The system significantly reduced safety critical errors and increased the accuracy of the rehabilitation relevant sequence. A key outstanding question was the extent to which Guide generalises to other tasks with different structure. For example, donning a prosthetic limb is mainly a procedural task, but would Guide also be able to support tasks which have a larger motivational component? Accordingly, the current study presents data on the effectiveness of Guide to support the morning routine, a complex sequence with both procedural and motivational aspects. Guide has previously demonstrated efficacy in an in patient/hospital setting with persons aiming to learn a rehabilitation task. This study also examines whether Guide can be an effective support for independence in the less structured setting of the participants own home post discharge. 


\section{Methodology}

Settings

1. A neurobehavioural assessment and rehabilitation unit specialised in the care of persons with complex needs after brain injury. 2. Service user's home.

\section{Design}

A single participant A-B-A'-B'-A"'-B" design (see Table 1) was used to assess the effectiveness of Guide to support the morning routine of a gentleman with 1 year history of severe haemorrhagic brain injury.

Outcome variables were number of errors made in the sequence and number of interventions required by rehabilitation support workers to ensure sequence performance.

Following discharge his performance with and without Guide was also assessed via ratings made by a family member. Thus, the usability and effectiveness in a domestic context was also assessed.

\section{Participant}

$\mathrm{Mr} \mathrm{M}$ is a 61 year old single retired electrician. He had one adult daughter who was not a dependent. He was found unconscious at his home after a presumed collapse. Admission Glasgow Coma Scale was 3/15 and brain imaging revealed a right intracerebral haemorrhage affecting the territory of the caudate nucleus and extending into the $3^{\text {rd }}$ and $4^{\text {th }}$ ventricles. Hydrocephalus and midline shift led to the insertion of an extra ventricular drain. Initial GCS and duration of PTA placed him in an extremely severe category of brain injury.

Mr M was admitted to a neurorehabilitation centre at 10 weeks post injury. Neuropsychological report at 14 weeks indicated that he had severe impairment of memory, executive function and visual perception. Impairment 
of verbal recall of information was associated with judgement difficulties. He lacked capacity to make legal or financial decisions as a result. He did not exhibit challenging behaviour and his mood was euthymic for the most part. Some anxiety was apparent if disoriented to place. Anxiety was also thought to have had a negative bearing on his sleep pattern and he required zopiclone to aid sleep onset. There was no evidence of significantly altered personality or current mood issues.

Medication remained constant throughout the trial and comprised: amlodipine, ramipril, ibuprofen, trazodone, co-codamol, lactulose, zopiclone, omeprazole, and eumovate.

Mr $\mathrm{M}$ was referred to the current project as he was identified by his Occupational Therapist staff as having difficulty performing his morning routine, improved by the verbal prompts of rehabilitation support workers.

\section{Materials}

Dell, Precision M4500 laptop.

Guide software loaded with morning routine protocol (appendix 1).

Acoustic Magic, Voicetracker2 array microphone.

Creative, Inspire T10 speakers.

Morning Checklist (Appendix 2) - Rating measure designed to quantify performance of morning personal care routine.

\section{Procedure}

Mr M was assessed by his Occupational Therapist who noted omissions of parts of the morning routine in the context of verbal report that he had completed those steps. The Morning Checklist (Appendix 2) was then introduced, to be completed by Rehabilitation Support Workers each morning. The observer rated the steps in the sequence as follows: completes step independently (5 points); completes step after 1 verbal prompt (4); completes step after 2 verbal prompts (3); completes step after 3 verbal prompts (2); requires physical intervention/assistance to start, continue or complete step 
(1); refuses to complete step (0 points). Only prompts by human carers were scored, prompts by the assistive technology (Guide) were not.

Following each morning routine performance in both baseline and intervention, the participant was asked to rate "how well did that go?" along a 5 point Likert scale from 5 "very well" to " 1 very poorly". This was with the aim of tapping into his perspective on his own behavioural performance and the support that he received.

Suitability for a trial of Guide was decided by the Occupational Therapist. The functionally defined inclusion criteria were that he was able to carry out sequences without errors or omissions when given verbal prompts by the Rehabilitation Support Workers, but omitted steps when acting independently.

After a baseline $(A)$ period of six weeks, giving 28 datapoints, Guide was installed in his bedroom to automatically activate at 8am and continue until morning routine was complete. The Guide was activated and data was recorded only on week days (Monday to Friday). The voice used in Guide was female $(\mathrm{CB})$ and familiar to $\mathrm{Mr} \mathrm{M}$. There were 12 datapoints in the intervention period 3 weeks and some missing datapoints. Rehabilitation Support Workers continued ratings in the Guide intervention (B) period Guide was in use. They were asked to prompt if there was a safety critical error or omission of an important sub-step.

The Guide was inadvertently switched off after week 6 of the intervention leading to a de facto return to baseline inpatient phase of 4 days ( $A^{\prime}$ - 3 data points). Guide was then reactivated and a second inpatient intervention phase of 4 weeks (B'-11 data points) took place.

Following agreement from the multidisciplinary team and community support agencies, Mr M was discharged to his own home (29.6.12). In this domestic situation the morning routine performance was assessed using the Morning Checklist, completed by Mr M's sister. Rightful assertion of privacy and 
variable visiting times meant that direct observations were not possible as in the rehabilitation centre.

The domestic install had a baseline of 6 data points and an intervention phase of 11 data points. Findings are reported under in-patient and home headings.

\section{Data analysis}

The single case study data were analysed using the Non-overlap All Pairs method (NAP: Parker and Vannest 2009, Parker et al. 2011). NAP represents a new application of established statistical methods, known variously as Area Under the Curve, Mann Whitney's $U$ and dominance statistics, to single subject designs. NAP has been demonstrated to be superior to other non overlap techniques in its precision, discrimination and relationship to established effect size measures such as R2 (Parker and Vannest, 2009). An assumption of the method is that there is no underlying trend in the baseline data. The Mann Kendall test of trend (Onoz and Bayazit, 2003) was nonsignificant $p>0.05$ ( 2 tailed) for the baseline period. To further explore stability we also examined the regression equations for the baseline data. A cubic equation had a higher R2 (0.168) and accounted for more of the variance than the linear model. This indicated that baseline scores fluctuated rather than gradually improved. Parker and Vannest (2009) give approximate values for evaluating effect sizes based on the NAP statistic: weak effects 0-0.65, medium effects $0.66-0.92$, large or strong effects $0.93-1.0$..

\section{Findings}

In-patient

During the first intervention phase the Morning Checklist score was significantly increased in comparison with baseline (Nonoverlap All Pairs = 0.754). This represents a medium effect size (Parker and Vannest, 2009). This indicates that the number of prompts given by support staff significantly decreased in the first intervention phase. In the B' phase the participant approached perfect performance (4.94/5.00). 
The participant was rated to have completed all the steps without prompt only once in the baseline period (3.6\%) and completed the sequence independently 6 times out of 12 datapoints (50\%) during the first intervention period. Visual presentation of these changes in performance can be seen in Figure 1.Given the short duration of the return to baseline phase $\left(A^{\prime}=3\right.$ data points) comparisons based solely on this data are likely to lack statistical power. If however the inpatient data are combined, comparing all inpatient intervention data $\left(B+B^{\prime}\right)$ to all inpatient control data $\left(A+A^{\prime}\right)$ gives a NAP statistic of 0.804 (a medium effect size Parker and Vannest, 2009).

[Insert Table 1 about here]

Sequence performance errors were significantly reduced during intervention phase (See Figure 2). The participant made on average 1.04 (Standard deviation 1.23) errors in baseline (A) and an average of 0 (Standard Deviation $=0$ ) in the first intervention (B) phase. This indicates that, for this participant, Guide use reduced errors to near zero. During the intervention period in the rehabilitation centre, there did not appear to be a habituation effect such that performance was constant throughout the intervention period, dipping only on return to baseline.

Home

This pattern of improvement was also apparent at home. The Non overlap all pairs (A"'-B') $=0.74$, medium effect size (Parker and Vannest, 2009) indicating a significant improvement in morning checklist ratings from baseline and intervention conditions (A"'-B'). Figure 1 shows both in-patient and home prompt scores.

[Insert Figure 1 about here]

On returning home without Guide (A") there was an increase in the error score to an average of 1.67 (sd 1.75). This resolved once Guide was 
activated (average B"=1.36 sd 0.67) but error rates were not reduced to zero, at least in the first ten days. This may have been an artefact of difference in observers or due to the change of environment.

In the inpatient baseline phase (A) the participant made errors of: getting up and going straight back to bed, hesitating during sequence, not being able to find clothes that are in the room, and not getting all the clothes ready to be fully dressed. After discharge (A") the more frequent errors were: not getting all clothes to be fully dressed, wearing dirty or mismatched clothes, forgetting to pick up phone/GPS and inappropriate clothes for the weather.

[Insert Figure 2 about here]

\section{Personal preference}

The mean rating of how the morning routine went was 4.5 in the baseline phase and 4.33 in the intervention phase (non significant). Both mean scores of satisfaction lay in the range between "very well" and "quite well". Mr M did not have a clear preference for either support. There was a slight anomaly in these findings as Mr M reported not wishing to have rehabilitation support workers support him on several occasions.

\section{Implications}

The prompting technology, Guide, reduced the participants errors to near zero in the intervention phase of the study and led to ratings of 'independent' on half of intervention trials. This trial thus evidences that Guide can emulate the supportive action of carers in the performance of a complex ADL sequence.

The implications for practice of the use of this technology encompass the augmentation of rehabilitation, facilitating discharge and home support. In terms of its use a rehabilitation tool, Guide might augment the amount of prompting support for a specific routine without increasing demands on staff. 
Rehabilitation Support Workers might then be freed to address the emotional and motivational needs of those not-responding to automated prompting.

Many service users object to being observed and prompted. The use of an automated, non-judgemental and emotionally neutral prompter may be preferable to those who find the interpersonal act of being prompted noxious (O’Neill \& Gillespie, 2008; Lo Presti et al., 2004). Such technologies might therefore increase the user's independence in ADL sequences such as the morning routine.

The technology may aid in the transition from rehabilitation centre to home. For example, if the person can carry out the sequence with prompting support, then Guide or a context aware prompter may function as a cognitive prosthetic and allow that person to be independent. Self-neglect is a common consequence of cognitive impairments. This case study raises the intriguing possibility that tendency to omit personal care regimens may be offset by context aware prompters.

Context aware prompting technologies are novel but are gaining an evidence base for their effectiveness to support independent activity. There are no commercially available auditory verbal context aware prompting systems.

There are limitations to the design reported. The participant was previously interested in technologies and may have been more compliant as a result. $\mathrm{He}$ was also perhaps motivated by a wish to be discharged to his own home.

Contemporary single $n$ experimental designs also include a variable which is not expected to change in response to the intervention, omitted from this experimental design.

Increases in error rates and decreases in prompt score ratings of independence occurred on return home and baseline. The transition to home was prepared for by many supported visits and overnight passes. However 
the emotional and cognitive demands of operating in a new environment may have acted to increase errors.

Future studies might confirm these findings outside of the originating research group who are currently recruiting for a randomised control trial due to report in 2014. Single n or case control methodologies would be suitable and Guide software would be available to such investigators.

\section{Acknowledgments}

This work was, in part, possible by Grant $\mathrm{CZH} / 4 / 598$ of the Chief Scientist Office of Health Service Directorate of the Scottish Government which is funding a randomised control trial of the technology. This trial is hosted by the Brain Injury Rehabilitation Trust and we are grateful to all staff who have contributed to the project. We are grateful to $\mathrm{Mr} \mathrm{M}$ for trialling the system and to his family in supporting a trial home installation. Guide is a prototype system and is not commercially available.

\section{References}

Aunger, R., (2010). Types of technology. Technological Forecasting and Social Change, Vol.77 No.5, pp.762-782.

Clarke, A., (2003). Natural-Born Cyborgs: Minds, Technologies, and the Future of Human Intelligence, Oxford University Press: Oxford.

Cole, E. (1999). Cognitive prosthetics: An overview to a method of treatment. NeuroRehabilitation, Vol.12, pp.39-51.

Davies, D. K., Stock, S. E., \& Wehmeyer, M. L. (2002). Enhancing independent task performance for individuals with mental retardation through use of a handheld self-directed visual and audio prompting system. Education and Training in Mental Retardation and Developmental Disabilities, Vol. 37, No. 2 pp. 209-218. 
Gillespie, A., Best, C., O’Neill, B. (2012) Cognitive function and assistive technology for cognition: A systematic review, Journal of the International Neuropsychological Society, Vol. 18 No.1, 1-19.

Kim, H. J., Burke, D. T., Dowds Jr., M. M., Boone, K. A. R., \& Park, G. J. (2000). Electronic memory aids for outpatient brain injury: follow-up findings. Brain Injury, Vol. 14, No. 2 pp.187-196.

Kirsch, N L, Shenton, M, \& Rowan, J. (2004). A generic, "in-house", alphanumeric paging system for prospective activity impairments after traumatic brain injury. Brain Injury, Vol.18, No. 7 pp.725-734.

Lancioni, Giulio E., O’Reilly, M. F., Seedhouse, Philip, Furniss, Frederick, \& Cunha, Bernardo. (2000). Promoting independent task performance by persons with severe developmental disabilities through a new computer-aided system. Behavior Modification, Vol. 24, No.5 pp700 -718.

Leirer, V. O., Morrow, D. G., Tanke, E. D., \& Pariante, G. M. (1991). Elders' nonadherence: its assessment and medication reminding by voice mail. The Gerontologist, Vol. 31, No. 4 pp514-520.

LoPresti, E.F., Mihailidis, A. \& Kirsch, N., (2004). Assistive technology for cognitive rehabilitation: State of the art. Neuropsychological Rehabilitation, Vol.14 No.1/2, pp.5-39.

Mihailidis, A., Barbenel, J. C., \& Fernie, G. (2004). The efficacy of an intelligent cognitive orthosis to facilitate handwashing by persons with moderate to severe dementia. Neuropsychological Rehabilitation, Vol.14, pp135-171.

Mihailidis, A., Boger, J., Craig, T., \& Hoey, J. (2008). The COACH prompting system to assist older adults with dementia through handwashing: An efficacy study. BMC Geriatrics, Vol.8, pp28. 
Oddy, M., and Worthington, A., (2009). The Rehabilitation of Executive Disorders: A Guide to Theory and Practice. Oxford University Press: Oxford

Parker, R. and Vannest, K. (2009). An improved effect size for single $n$ research: Non-overlap of All Pairs. Behavior Therapy, Vol.40, No. 4 pp357367

Parker, R.I., Vannest, K.J. and Davis, J.L., (2011). Effect Size in Single-Case Research: A Review of Nine Nonoverlap Techniques. Behavior Modification, Vol. 35 No.4, pp.303 -322 .

Pijnenborg, G. H. M., Withaar, F. K., Evans, J. J., van den Bosch, R. J., \& Brouwer, W. H. (2007). SMS text messages as a prosthetic aid in the cognitive rehabilitation of schizophrenia. Rehabilitation Psychology, Vol.52, No. 2 pp236-240.

Svoboda, E., \& Richards, B. (2009). Compensating for anterograde amnesia: a new training method that capitalizes on emerging smartphone technologies. Journal of the International Neuropsychological Society, Vol. 15,No.4 pp629638.

van den Broek, M. D. V. D., Downes, J., Johnson, Z., Dayus, B., \& Hilton, N. (2000). Evaluation of an electronic memory aid in the neuropsychological rehabilitation of prospective memory deficits. Brain Injury, Vol.14, No. 5 pp455-462.

Wilson, B. A., Emslie, H. C., Quirk, K., \& Evans, J. J. (2001). Reducing everyday memory and planning problems by means of a paging system: $\mathrm{A}$ randomised control crossover study. Journal of Neurology Neurosurgery and Psychiatry, Vol. 70, No. 4 pp477-482.

World Health Organization. (2002). Towards a common language for functioning, disability and health (ICF). Geneva. Available at 
http://www.who.int/classifications/icf/training/icfbeginnersguide.pdf (accessed 28th August 2012) 
Table 1 Morning Routine Performance by Phase

\begin{tabular}{|l|l|l|l|l|l|l|}
\hline Setting & Phase & $\begin{array}{l}\text { Total no. } \\
\text { of days }\end{array}$ & $\begin{array}{l}\text { Data } \\
\text { points }\end{array}$ & $\begin{array}{l}\text { Mean } \\
\text { Morning } \\
\text { Checklist } \\
\text { score (sd) }\end{array}$ & $\begin{array}{l}\text { Days fully } \\
\text { independent } \\
(\%)\end{array}$ & $\begin{array}{l}\text { Mean error } \\
\text { score (sd) }\end{array}$ \\
\hline \multirow{2}{*}{$\begin{array}{l}\text { In- } \\
\text { patient }\end{array}$} & Baseline-A & 30 & 28 & $4.49(0.28)$ & $1(3.57)$ & $1.04(1.23)$ \\
\cline { 2 - 7 } & Intervention_B & 31 & 12 & $4.75(0.04)$ & $6(50)$ & $0(0)$ \\
\cline { 2 - 7 } & $\begin{array}{l}\text { Return to } \\
\text { baseline-A' }\end{array}$ & 4 & 3 & $4.58(0.72)$ & $2(66.67)$ & $0.33(0.58)$ \\
\cline { 2 - 7 } & Intervention- B' & 20 & 11 & $4.94(0.13)$ & $8(72.7)$ & $0.09(0.30)$ \\
\hline \multirow{2}{*}{ Home } & Baseline-A" & 6 & 6 & $4.43(0.78)$ & $0(0)$ & $1.67(1.75)$ \\
\cline { 2 - 7 } & Intervention-B" & 14 & 11 & $4.8(0.19)$ & $2(18.18)$ & $1.36(0.67)$ \\
\hline
\end{tabular}

Figure 1 Morning checklist score by phase

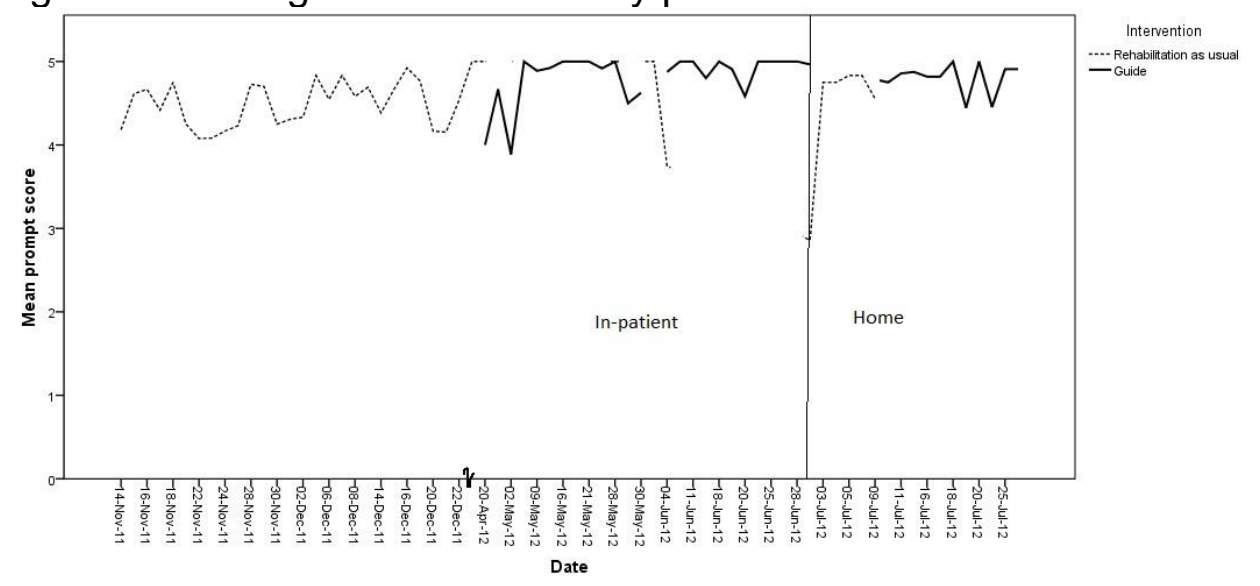

Figure 2 Performance errors by phase

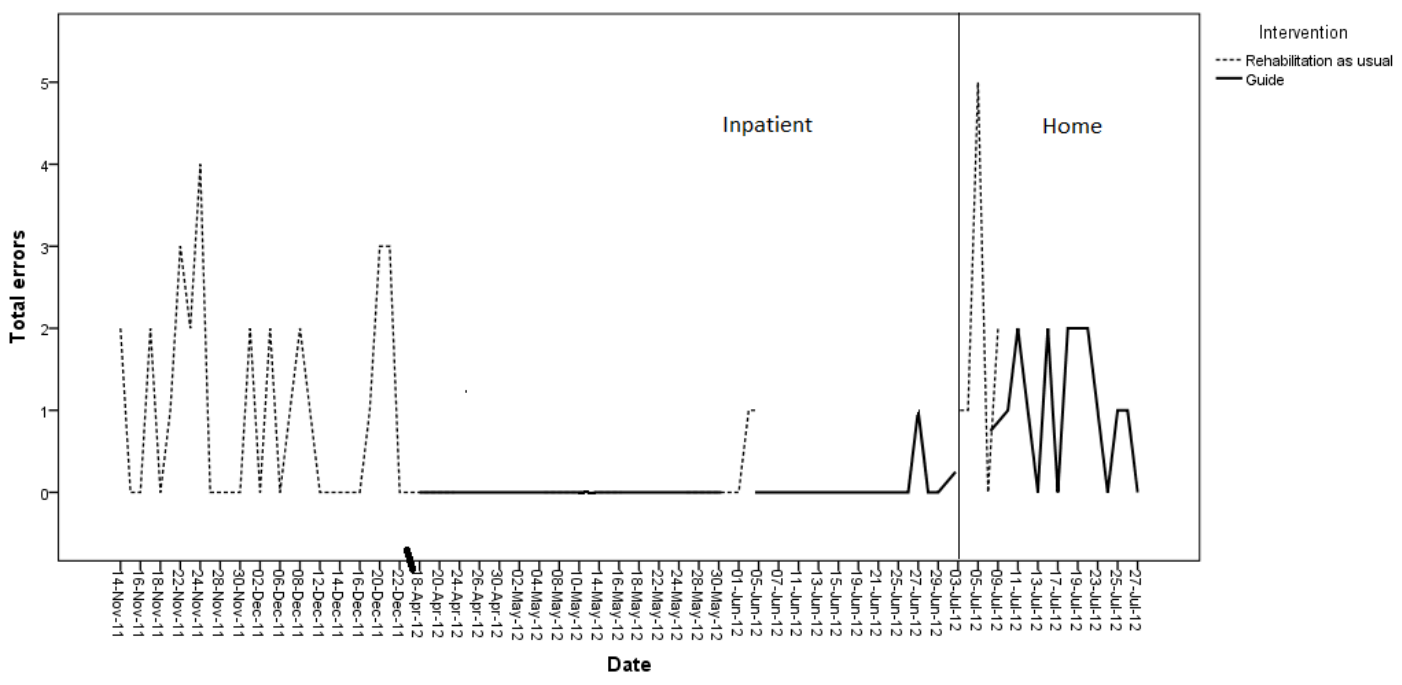





\section{Appendix 1 \\ Morning routine protocol}

P1. Good morning it is 8 o'clock..... nearly time to get up. (5mins break)(WAKE UP STEP)

P1.1: Good morning it is five past eight. Nearly time to get up and have some breakfast. (5mins)

P1.2 Morning soon it will be time to get up and have a shower before you go down for breakfast. (5 mins)

P1.3 Ok now its time to get up (GET UP STEP)

Q1.4 Have you got out of bed yet? N \& NR=Q1.5 $Y=P 3$

Q1.5: Are you tired? Would you like five more minutes to wake up? $Y \& N R=P 1.6$, $\mathrm{N}=\mathrm{Q} 1.8$

P1.6 OK (5 mins)

P1.7 Its now twenty past eight time to get up. Q1.9

P1.8 Then its time to get up.

Q1.9 Have you got out of bed? $Y=P 3, N$ \& $N=Q 2$

$\mathrm{Q} 2$ Would you like to have some breakfast? $\mathrm{Y}=\mathrm{Q} 2.1 \mathrm{~N}=\mathrm{Q} 2.3$

P2.1 If you get up now you will be in time for breakfast.

Q2.2 Have you got out of bed now? $Y=P 3 N=Q 2.6$

$\mathrm{Q} 2.3$ If you don't have breakfast do you get hungry before lunchtime? $Y=Q 2.3 .1$

$\mathrm{N}=\mathrm{Q} 2.4$

Q2.3.1 Do you want to be up before breakfast finishes? $Y=P 2.1 \mathrm{~N} \& \mathrm{NR}=\mathrm{Q} 2.4$

Q2.4: Would you like some nice hot coffee? N \& NR=Q2.5 Y=P2.4.1

P2.4.1 Get up soon to make sure you are in time for coffee. Q2.2

Q2.5 Do you like to get up independently? N \& NR= P2.6 Y=P2.5.1

P2.5.1 If you get up now you can show you can do it on your own.

P2.6 Its time to get out of bed

Q2.7 Have you got out of bed? N \& NR=P2.7 Y=P3

P3 OK before going down for breakfast you need to have a shower. (MOTIVATE SHOWER STEP)

Q3.1 Are you going to get into the shower now? N \& NR=Q3.2 Y=P4

Q3.2 Do you feel hot and sticky? $Y=P 3.2 .2 \mathrm{~N} \& \mathrm{NR}=P 3.2 .1$

P3.2.1 Even if you don't feel too bad having a shower will make you feel fresh and clean.Q3.3

P3.2.2 Having a shower will make you feel fresh and clean.

Q3.3 Are you going to get into the shower? $Y=P 4 N$ \& NR=Q3.4

Q3.4 Do your friends and family think you keep yourself clean and tidy? Y=P3.4.1 N

$\& N R=Q 3.5$

P3.4.1 Having a shower will make sure that you look clean and fresh.

Q3.5 Are you someone who likes to look clean and presentable? $Y=Q 3.3 N=Q 3.6$

Q3.6 Do you like to do things for yourself without anyone having to remind you?

$\mathrm{N}=\mathrm{Q} 3.7 \mathrm{Y}=\mathrm{P} 3.6 .1$

P3.6.1 If you have a shower without prompting it will show you can do it yourself.

Q3.6.2 Are you going to have a shower now? N=Q3.7 $\mathrm{Y}=\mathrm{P} 4$

Q3.7 Do you want to move out of [residential home] to live somewhere more independent? N=P3.8 Y=P3.7.1

P3.7.1 Getting up and having a shower by yourself shows that you are ready to be more independent.

P3.8 You need to have a shower before going down to the communal areas.

Q3.9 Are you going to have a shower?

P4. That's great. Now you need to get together everything you need for a shower. (SHOWER THINGS STEP)

Q4.1 Have you got a towel? $\mathrm{N}=\mathrm{Q} 4.1 .1 \mathrm{Y}=4.2$

Q4.1.1 Is there a towel in your room? N=P4.1.2 $Y=Q 4.2$ 
P4.1.2 Then we need to get some help.

Q4.2 Have you got some shower gel? N=Q2.3 $\mathrm{Y}=\mathrm{P} 2.4$

Q2.3 Can you see some shower gel in the bathroom? N=P2.3.1 Y=P2.4

P2.3.1 The take some soap with you into the shower

Q2.4 Have you got some shampoo? Y=P2.5

Q2.4.1 Can you see any shampoo in the bathroom? $Y=P 2.5 \mathrm{~N}=P 2.4 .2$

P2.4.2 Then use shower gel to wash your hair.

P2.4.3 Don't forget to put your night clothes into the laundry basket.

P2.5 Good. You are ready for your shower.

P3.1 Have you got out of the shower? N \& NR=Q3.2 $Y=Q 5.3$

Q3.2 Are you out of the shower now? N \& NR=Q3.1 $Y=Q 3.2$

P3.2 Good. Now you need to get yourself looking clean and smart for the day ahead (DRY OFF STEP)

Q3.3 Have you shaved this morning? $Y=Q 3.5 \mathrm{~N} \& \mathrm{NR}=\mathrm{P} 3.4$

P3.4 You need to have a shave every day.

Q3.5 Are you going to have a shave now? Y=P3.6 N=Q3.9

P3.6 Don't forget to use shaving foam and a new razor. (5mins)

Q3.7 Have you finished your shave? N \& NR=Q3.8 $Y=Q 3.9$

Q3.8 Have you finished shaving? N \& NR=Q3.7 $Y=Q 3.9$

Q3.9 Have you cleaned your teeth? $Y=P 4.1 \mathrm{~N}=\mathrm{P} 3.9 .1$

P3.9.1 Clean your teeth before you go down for breakfast (2mins)

P4.1 Before you get dressed you need to make sure you are completely dry.

Q4.2 Have you dried yourself all over? Y, N \& NR=4.3

Q4.3 Have you dried your back and your legs? $N=P 4.4 Y=4.5 \mathrm{NR}=\mathrm{R}$

P4.4 Make sure you dry yourself thoroughly.

Q4.5 Have you put on some deodorant? N \& NR= P4.6 Y=P5

P4.6 Put on some deodorant before getting dressed.

P5 Great. Now you need to think about what clothes to wear today. (CHOOSE CLOTHES STEP)

Q5.1 Do you need to look smart today? NR=Q5.2 $Y \& N=P 5.3$

Q5.2 Have you got any meetings or are you going out somewhere? $Y, N$ \&NR=P5.3

P5.3 Also think about the weather. Is it particularly cold or rainy today? Y, N

$\& N R=P 5.4$

P5.4 Now choose some suitable clothes.

Q5.5 Do the clothes you have chosen match? $\mathrm{Y}=\mathrm{P} 6 \mathrm{~N} \& \mathrm{NR}=\mathrm{P} 5.51$

P5.5.1 Choose some clothes that look good together.

P6 Right. Now put on your clothes.

P7 Okay the next step is to check that you are ready for the day ahead. (READY FOR DAY STEP)

Q6.1 Have you made your bed? N \& NR=P6.1.1 $Y=Q 6.2$

P6.1.1 Then put the duvet straight.

Q6.2 Have you got your mobile phone? N \& NR=P6.3.1 $Y=Q 6.7$

P6.3.1 Then take your mobile phone with you.

P7 That's great. You are all set for the day. 
Appendix 2

Morning Checklist

CLIENT NAME:

W/C:

Level of prompting

\begin{tabular}{|l|l|l|l|l|l|l|l|}
\hline & M & T & W & T & F & S & S \\
\hline Wake up & & & & & & & \\
\hline Get out of bed & & & & & & & \\
\hline Use toilet & & & & & & & \\
\hline Wash hands & & & & & & & \\
\hline Go into shower & & & & & & & \\
\hline Shower: Wash upper half & & & & & & & \\
\hline \multicolumn{1}{|c|}{ Wash lower half hair } & & & & & & & \\
\hline Brush teeth & & & & & & & \\
\hline Dry self & & & & & & & \\
\hline Shave: Wet / Dry & & & & & & & \\
\hline Use deodorant & & & & & & & \\
\hline Select appropriate clothes & & & & & & & \\
\hline Find clothes & & & & & & & \\
\hline Dress & & & & & & & \\
\hline Brush hair & & & & & & & \\
\hline Make bed & & & & & & & \\
\hline Medication prompt by staff Y/N & & & & & & & \\
\hline Picks up phone/keys/cigarettes & & & & & & & \\
\hline $\begin{array}{l}\text { Rating of personal appearance } \\
\text { (out of 10) }\end{array}$ & & & & & & & \\
\hline Time up & & & & & & & \\
\hline Completed by: & & & & & & \\
\hline
\end{tabular}

$5=$ Completes step independently

4 = Completes step after 1 verbal prompt

$3=$ Completes step after 2 verbal prompts

2 = Completes step after 3 verbal prompts

1 = Requires physical intervention / assistance to start, continue or complete step

$\mathrm{R}=$ Refuses to complete step.

$\mathrm{N} / \mathrm{E}=$ No evidence

$\mathrm{N} / \mathrm{A}=$ Not appropriate (e.g. woman who does not shave)

Errors (circle Y / N)

\begin{tabular}{|l|c|c|c|c|c|c|c|}
\hline & M & T & W & T & F & S & S \\
\hline Stays in bed until after 10am & $\mathrm{Y} / \mathrm{N}$ & $\mathrm{Y} / \mathrm{N}$ & $\mathrm{Y} / \mathrm{N}$ & $\mathrm{Y} / \mathrm{N}$ & $\mathrm{Y} / \mathrm{N}$ & $\mathrm{Y} / \mathrm{N}$ & $\mathrm{Y} / \mathrm{N}$ \\
\hline Gets up but goes straight back to bed & $\mathrm{Y} / \mathrm{N}$ & $\mathrm{Y} / \mathrm{N}$ & $\mathrm{Y} / \mathrm{N}$ & $\mathrm{Y} / \mathrm{N}$ & $\mathrm{Y} / \mathrm{N}$ & $\mathrm{Y} / \mathrm{N}$ & $\mathrm{Y} / \mathrm{N}$ \\
\hline Does not take towel to shower & $\mathrm{Y} / \mathrm{N}$ & $\mathrm{Y} / \mathrm{N}$ & $\mathrm{Y} / \mathrm{N}$ & $\mathrm{Y} / \mathrm{N}$ & $\mathrm{Y} / \mathrm{N}$ & $\mathrm{Y} / \mathrm{N}$ & $\mathrm{Y} / \mathrm{N}$ \\
\hline
\end{tabular}




\begin{tabular}{|l|c|c|c|c|c|c|c|}
\hline \hline Does not take soap /shower gel to shower & $\mathrm{Y} / \mathrm{N}$ & $\mathrm{Y} / \mathrm{N}$ & $\mathrm{Y} / \mathrm{N}$ & $\mathrm{Y} / \mathrm{N}$ & $\mathrm{Y} / \mathrm{N}$ & $\mathrm{Y} / \mathrm{N}$ & $\mathrm{Y} / \mathrm{N}$ \\
\hline & $\mathrm{M}$ & $\mathrm{T}$ & $\mathrm{W}$ & $\mathrm{T}$ & $\mathrm{F}$ & $\mathrm{S}$ & $\mathrm{S}$ \\
\hline $\begin{array}{l}\text { Does not get all the clothes necessary to be fully } \\
\text { dressed }\end{array}$ & $\mathrm{Y} / \mathrm{N}$ & $\mathrm{Y} / \mathrm{N}$ & $\mathrm{Y} / \mathrm{N}$ & $\mathrm{Y} / \mathrm{N}$ & $\mathrm{Y} / \mathrm{N}$ & \multicolumn{2}{|c|}{$\mathrm{PTO}$} \\
\hline Does not take shampoo & $\mathrm{Y} / \mathrm{N}$ & $\mathrm{Y} / \mathrm{N}$ & $\mathrm{Y} / \mathrm{N}$ & $\mathrm{Y} / \mathrm{N}$ & $\mathrm{Y} / \mathrm{N}$ & $\mathrm{Y} / \mathrm{N}$ & $\mathrm{Y} / \mathrm{N}$ \\
\hline Cannot find an item of clothing that is in the room & $\mathrm{Y} / \mathrm{N}$ & $\mathrm{Y} / \mathrm{N}$ & $\mathrm{Y} / \mathrm{N}$ & $\mathrm{Y} / \mathrm{N}$ & $\mathrm{Y} / \mathrm{N}$ & $\mathrm{Y} / \mathrm{N}$ & $\mathrm{Y} / \mathrm{N}$ \\
\hline Dresses when still wet & $\mathrm{Y} / \mathrm{N}$ & $\mathrm{Y} / \mathrm{N}$ & $\mathrm{Y} / \mathrm{N}$ & $\mathrm{Y} / \mathrm{N}$ & $\mathrm{Y} / \mathrm{N}$ & $\mathrm{Y} / \mathrm{N}$ & $\mathrm{Y} / \mathrm{N}$ \\
\hline Once out of bed hesitates for 3+ seconds & $\mathrm{Y} / \mathrm{N}$ & $\mathrm{Y} / \mathrm{N}$ & $\mathrm{Y} / \mathrm{N}$ & $\mathrm{Y} / \mathrm{N}$ & $\mathrm{Y} / \mathrm{N}$ & $\mathrm{Y} / \mathrm{N}$ & $\mathrm{Y} / \mathrm{N}$ \\
\hline Inappropriate clothes chosen for weather & $\mathrm{Y} / \mathrm{N}$ & $\mathrm{Y} / \mathrm{N}$ & $\mathrm{Y} / \mathrm{N}$ & $\mathrm{Y} / \mathrm{N}$ & $\mathrm{Y} / \mathrm{N}$ & $\mathrm{Y} / \mathrm{N}$ & $\mathrm{Y} / \mathrm{N}$ \\
\hline Dirty /mismatched clothes worn & $\mathrm{Y} / \mathrm{N}$ & $\mathrm{Y} / \mathrm{N}$ & $\mathrm{Y} / \mathrm{N}$ & $\mathrm{Y} / \mathrm{N}$ & $\mathrm{Y} / \mathrm{N}$ & $\mathrm{Y} / \mathrm{N}$ & $\mathrm{Y} / \mathrm{N}$ \\
\hline Poor personal hygiene & $\mathrm{Y} / \mathrm{N}$ & $\mathrm{Y} / \mathrm{N}$ & $\mathrm{Y} / \mathrm{N}$ & $\mathrm{Y} / \mathrm{N}$ & $\mathrm{Y} / \mathrm{N}$ & $\mathrm{Y} / \mathrm{N}$ & $\mathrm{Y} / \mathrm{N}$ \\
\hline Unshaven & $\mathrm{Y} / \mathrm{N}$ & $\mathrm{Y} / \mathrm{N}$ & $\mathrm{Y} / \mathrm{N}$ & $\mathrm{Y} / \mathrm{N}$ & $\mathrm{Y} / \mathrm{N}$ & $\mathrm{Y} / \mathrm{N}$ & $\mathrm{Y} / \mathrm{N}$ \\
\hline Forgets phone/keys/cigarettes & $\mathrm{Y} / \mathrm{N}$ & $\mathrm{Y} / \mathrm{N}$ & $\mathrm{Y} / \mathrm{N}$ & $\mathrm{Y} / \mathrm{N}$ & $\mathrm{Y} / \mathrm{N}$ & $\mathrm{Y} / \mathrm{N}$ & $\mathrm{Y} / \mathrm{N}$ \\
\hline
\end{tabular}

\section{Sequence errors}

\begin{tabular}{|l|l|l|l|l|l|l|l|}
\hline & M & T & W & T & F & S & S \\
\hline No of times repeats a step & & & & & & & \\
\hline No of steps missed & & & & & & & \\
\hline No of times stuck on a step & & & & & & & \\
\hline Time taken & & & & & & & \\
\hline
\end{tabular}

\section{Service user satisfaction (1-5)}

How well did that go?
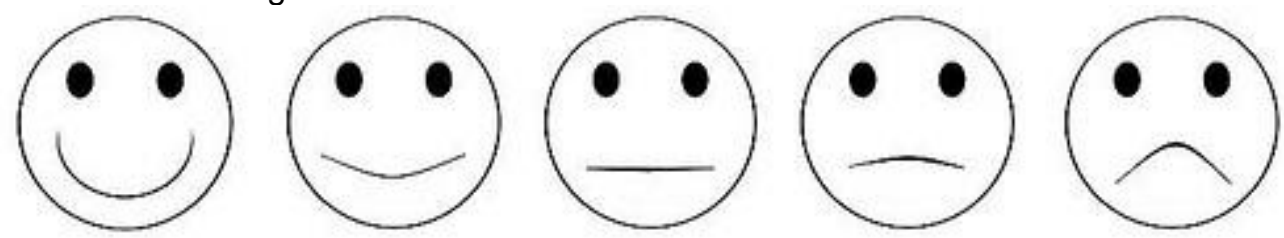

5 Very well

4 Quite well

$3 \mathrm{Ok}$

2 Quite poorly 1 Very poorly

\begin{tabular}{|l|l|l|l|l|l|l|l|}
\hline & M & T & W & T & F & S & S \\
\hline Rating & & & & & & & \\
& & & & & & & \\
\hline
\end{tabular}


